Check for updates

1 University of Leeds and Leeds Teaching and Community NHS Trusts, Leeds LS1 3EX, UK

2 Retired

3 Imperial College London, London, UK

Correspondence to: M Sivan M.Sivan@leeds.ac.uk

Cite this as: BMJ 2021;373:n853 http://dx.doi.org/10.1136/bmj.n853 Published: 01 April 2021

\section{Fresh evidence of the scale and scope of long covid}

\author{
The NHS must reconfigure services to manage enduring multimorbidity following covid-19
}

Manoj Sivan, ${ }^{1}$ Clare Rayner, ${ }^{2}$ Brendan Delaney ${ }^{3}$

The SARS-CoV-2 pandemic in the UK has so far affected more than four million people, with more than 450 ooo admitted to hospital and $30 \%$ in-hospital mortality. ${ }^{1}$ The UK's health service has risen to the twin challenges of managing waves of acute covid-19 and the vaccination effort, with rapid reconfiguration of both services and physical space. Beneath the daily headlines of admissions, deaths, and vaccinations is, however, hidden an iceberg of long term illness-known as long covid, post-covid syndrome, or post-covid sequelae.

In a linked paper, Ayoubkhani and colleagues (doi:10.1136/bmj.n693) extract data from all UK general practice records linked to hospital admissions to explore the risks of readmission, death, and incidence of respiratory, cardiovascular, metabolic, kidney, and liver diseases in a mean time frame of 140 days after discharge of patients admitted with covid-19. ${ }^{2}$ Strikingly, one third of discharged patients were readmitted to hospital and one in 10 died $(17.7 \%$ of those readmitted; D Ayoubkhani and A Banerjee, personal communication, 2021). Discharged patients had higher risks of new respiratory disease (6 times), major cardiovascular disease (3 times), chronic liver disease (2.8 times), chronic kidney disease (1.9 times), and diabetes (1.5 times) than matched controls from the general population. The risks were higher in those younger than 70 and in non-white individuals.

Another recent UK study showed that more than half of patients with covid-19 had long covid symptoms three months after discharge from hospital, with worse outcomes among those younger than 50 , women, and those with higher pre-covid fitness levels. ${ }^{3}$ Similar burdens of persistent symptoms and multiple organ impairment have been reported in studies from other countries ${ }^{4}$ and have also been observed in patients with covid-19 who were not admitted to hospital. ${ }^{6}$ Recent funding from the UK National Institute for Health Research (£20.om $(\$ 27.6 \mathrm{~m} ; € 23.4 \mathrm{~m})$ ) and the considerably more generous $\$ 1.15 \mathrm{bn}$ allocated in the US will help to characterise disease and potential mechanisms.

As our understanding of the scale and scope of long covid improves, it is clear that populations and the NHS will face a substantial burden of additional morbidity and long term conditions as a result of covid-19. The term long covid embraces a wide spectrum of organ involvement, with no clear evidence yet to help inform efficient diagnostic pathways or specific treatments or to indicate probable prognosis.

In addition to the respiratory, cardiac, renal, and endocrine pathology shown by this population level study and others, ${ }^{24}$ clusters of patients seem to have features of immunological dysfunction, dysautonomia, mast cell dysfunction, and neurological diagnoses. ${ }^{3}$ This is a problem for management, as common long covid symptoms (fatigue, cognitive dysfunction, wheeze, inappropriate tachycardia, gastrointestinal disturbance, for example) have multiple differential diagnoses spread across multiple specialties.

The NHS has always operated on a single specialty referral model, with general practice as the gatekeeper. With notable exceptions in elderly care, pain, and cancer services, hospital services are predominantly focused on individual organ systems. The longer term impact of covid-19 will require the NHS to rethink the way services are organised and delivered forpeople with long covid, including those presenting after discharge from hospital. Patients accessing care through their GP should expect careful history and examination, prompt baseline investigations, and the possibility of referral to one of the new multispecialty long covid clinics in a reasonable timeframe.

Early indications suggest that long covid clinics are often unavailable, might lack specialist medical supervision or specialist multidisciplinary teams, or have long waiting lists. Patients can present with symptoms requiring urgent assessment by specialists in integrated clinics (chest pain, breathlessness with desaturation on activity, cardiac arrhythmias).

Inappropriately lengthy or unidisciplinary pathways that lack the right skills to manage complexity are a risk to patient safety. Rehabilitation therapy teams need appropriate medical support and leadership, and GPs need to keep abreast of this rapidly evolving specialty. Listening to patients-who have often not been heard-is an important part of tackling inequality in access to healthcare and outcomes. ${ }^{8}$

As the NHS manages the proposed transition to integrated care systems, long covid is an urgent example of how new service delivery approaches must be designed to manage multimorbidity. Pathways must focus on capturing patients' multisystem symptoms and rehabilitation needs and provide individualised management programmes that aim for medical management and a return to normal functioning, including work. ${ }^{910}$ Although a place might exist for trials of specific treatments for some potential symptom clusters (eg, dysautonomia or mast cell disorder), the most pressing need is for rapid learning to understand what represents good multidisciplinary care, informed by real world outcome data and patient experience. ${ }^{11}$

Competing interests: The BMJ has judged that there are no disqualifying financia ties to commercial companies. The authors declare the following other interests: MS and colleagues developed the C19-YRS (Yorkshire rehabilitation scale) used for the assessment and monitoring of long covid symptoms; BD has received a speakers honorarium from Pfizer Oncology for speaking about real world data 
in cancer research. The BMJ policy on financial interests is here: https://www.bmj.com/sites/default/files/attachments/resources/2016/03/16-current-bmj-education-coi-form.pdf.

Provenance and peer review: Commissioned; not externally peer reviewed.

1 Coronavirus (COVID-19) in the UK. https://coronavirus.data.gov.uk/details/healthcare accessed 22.03.2021

2 Ayoubkhani D, Khunti K, Nafilyan V, etal. Post-covid syndrome in individuals admitted to hospital with covid-19: a retrospective cohort study. BMJ2021;372.

3 Sigfrid L, Drake TM, Pauley E, Jesudason EC, Olliaro P. Long Covid in adults discharged from UK hospitals after Covid-19: A prospective, multicentre cohort study using the ISARIC WHO Clinical Characterisation Protocol.medRxiv 2021.03.18.21253888.

4 Leung TYM, Chan AYL, Chan EW, etal. Short- and potential long-term adverse health outcomes of COVID-19: a rapid review. Emerg Microbes Infect 2020;9:2190-9. doi: 10.1080/22221751.2020.1825914 pmid: 32940572

5 Dennis A, Wamil M, Kapur S, etal. Multi-organ impairment in low-risk individuals with long COVID.medRxiv 202.12.08.20246025; doi: 10.1101/2020.12.08.20246025

6 Lambert N, Corps S, El-Azab SA, etal. COVID-19 survicors reports of the timing, duration, and health impacts of post-acute sequelae of SARS-CoV2 (PASC) infection.medRxiv 2021.03.22.21254026.

7 NHS England. National Guidance for post-COVID syndrome assessment clinics. www.england.nhs.uk/coronavirus/publication/national-guidance-for-post-covid-syndrome-assessmentclinics/ accessed 22.03.2021.

8 Altiery De Jesus VV, Alwan N, Callard F, Berger Z. Listening to Long COVID: Epistemic Injustice and COVID-19 morbidity.OSFPreprints 2021;0.31219/osf.io/tfbnd

9 Sivan M, Halpin SJ, Gee J. Assessing long-term rehabilitation needs in COVID-19 survivors using a telephone screening tool (C19-YRS tool). Adv Clin Neurosci Rehabil 2020;19:14-7doi: 10.47795/NELE5960.

10 Sivan M, Halpin S, Hollingworth L, Snook N, Hickman K, Clifton IJ. Development of an integrated rehabilitation pathway for individuals recovering from COVID-19 in the community. J Rehabil Med 2020;52:jrm00089.pmid: 32830284

11 Friedman CP, Allee NJ, Delaney BC, etal. The science of Learning Health Systems: Foundations for a new journal. Learn Health Syst 2016;1:e10020.pmid: 31245555

This article is made freely available for use in accordance with BMJ's website terms and conditions for the duration of the covid-19 pandemic or until otherwise determined by BMJ. You may use, download and print the article for any lawful, non-commercial purpose (including text and data mining) provided that all copyright notices and trade marks are retained. 